\title{
Détermination rapide et spécifique du taux d'hydrolyse du lactose par chromatographie liquide à haute performance à l'aide d'un étalon interne
}

\author{
par \\ M. DEMAIMAY* et C. BARON**
}

\section{INTRODUCTION}

Les procédés d'hydrolyses enzymatiques $[8,13,14,17,19]$ et catalytiques $[4,7]$ du lactose du lactosérum, qui peuvent conduire à des lactosérums concentrés hydrolysés ou à des concentrés de sucres liquides, ouvrent une voie intéressante pour la revalorisation de ce produit en alimentation humaine.

Ils nécessitent, tout particulièrement à l'échelon industriel, des techniques d'analyses qui permettent de contrôler rapidement l'évolution du taux d'hydrolyse, celui-ci étant défini par le rapport des concentrations glucose + galactose sur glucose + galactose + lactose.

Différentes méthodes sont actuellement utilisées pour sa détermination. Les plus pratiquées sont enzymatiques $[2,10]$, chimiques [18], et chromatographiques $[12,22]$. Elles présentent cependant le désavantage d'être longues et d'utilisation difficile sur le plan industriel.

L'analyse cryoscopique développée par Ramet [16] est mieux adaptée du fait de sa rapidité. Par contre, comme l'indique l'auteur, elle manque de spécificité chaque fois que la composition du milieu en matières solubles varie.

La chromatographie liquide à haute performance (CLHP) peut donc apporter de par sa spécificité et sa rapidité une solution intéressante à ce problème.

\footnotetext{
* Laboratoire de Chimie-Biochimie Alimentaire (E.N.I.T.I.A.A.), La Géraudière 44072 Nantes cedex.

** Laboratoire de Biochimie (E.N.S.B.A.N.A.), Campus Universitaire - 21000 Dijon.
} 
En effet, grâce à l'emploi de phase stationnaire de très faible granulométrie (5 à $10 \mu$ ), il est possible de préparer des colonnes chromatographiques très efficaces. La séparation des produits s'effectue à haute pression (jusqu'à 600 bars) par l'intermédiaire d'une pompe, afin de compenser les pertes de charge considérables qui sont dues aux faibles granulométries.

L'application de cette technique à l'analyse des sucres dans les milieux alimentaires a déjà été abordée par de nombreux auteurs $[1,3,5,9,11,15,20]$ tant sur le plan qualitatif que quantitatif, en particulier par Warthesen [21] et Euber [6] pour le dosage des sucres du lait et des desserts glacés.

Ici, nous proposons une méthode de contrôle rapide et spécifique de l'hydrolyse du lactose du lactosérum à l'aide de la chromatographie liquide à haute performance et nous en fixons les limites en la comparant à la méthode de dosage par chromatographie en phase gazeuse [22].

\section{MATERIELS ET METHODE}

\section{A. Echantillonnage :}

- lactosérum, déminéralisé, déprotéiné, hydrolysé par voie catalytique sur résines échangeuses d'ions et cristallisé ;

- lactosérum, déminéralisé, déprotéiné, hydrolysé sur enzyme fixée et concentré à $778 \mathrm{~g} / 1$;

- lactosérum non déprotéiné à $38,8 \mathrm{~g} / 1$ de sucres totaux.

B. Technique d'analyse des sucres par chromatographie liquide à haute performance

$1^{\circ}$ Appareillage

Colonne : Lichrosorb $\mathrm{NH}_{2} 5 \mu, 25 \times 4,6 \mathrm{~mm}$, Chrompack.

Un préfiltre est placé en tête de colonne.

Pompe : Pye Unicam LC 3-XP.

Injecteur : vanne à boucle de $10 \mu \mathrm{l}$, Valco.

Détecteur : réfractomètre différentiel Waters.

Enregistreur : Cole-Palmer Instrument Compagny.

Intégrateur : Système 1 autolab.

Seringue S.G.E. de $20 \mu \mathrm{l}$.

2० PRODUITS :

— solvant éluant : mélange (acétonitrile-eau distillée : 73-27) préalablement dégazé ;

- acétonitrile pour spectroscopie Merck ;

- D (+) glucose, D (+) galactose, lactose monohydraté Merck ; 
- étalon interne : raffinose pentahydraté Merck ;

- acide trichloracétique Merck ;

- papier filtre Whatman $n^{\circ} 42$.

3o PréPaRation des Échantillons

a) Hydrolysat de lactose pur et hydrolysat de lactose de lactosérum déprotéiné :

- si la concentration initiale en sucres totaux est inférieure ou égale à environ $50 \mathrm{~g} / \mathrm{l}$, ajouter l'étalon interne à raison de $20 \mathrm{~g} / \mathrm{l}$ avant l'injection ;

- si la concentration en sucres totaux est supérieure à $50 \mathrm{~g} / \mathrm{l}$, effectuer une dilution et ajouter l'étalon interne $(20 \mathrm{~g} / \mathrm{l})$ avant l'injection.

b) Hydrolysat de lactose de lactosérum non déprotéiné :

- si nécessaire ramener comme en $\S$ a) la concentration en sucres totaux au plus à $50 \mathrm{~g} / 1$;

- ajouter l'étalon interne ;

- ajouter ensuite de l'acide trichloracétique jusqu'à une concentration finale de 6 p. $100(\mathrm{P} / \mathrm{V})$. Laisser agir $5 \mathrm{~min}$, puis effectuer une filtration sur papier Whatman $n^{\circ} 42$. Cette opération est très rapide.

4० CONDITIONS OPÉRATOIRES

L'injection se fait en injectant $10 \mu$ l de l'échantillon dans la boucle de l'injecteur à l'aide de la seringue S.G.E.

Le débit du solvant éluant est fixé à $2,5 \mathrm{ml} / \mathrm{min}$.

La durée de la chromatographie est de $7 \mathrm{~min}$.

\section{Technique d'analyse des sucres par chromatographie en phase gazeuse}

\section{$1^{\circ}$ Appareillage}

Le chromatographe utilisé est un appareil Packard, modèle 419, équipé d'un détecteur à ionisation de flamme. L'intensité du courant dans le détecteur est de $2,5 \times 10^{-10} \mathrm{~A}$.

L'injection des échantillons se fait directement en tête de colonne à l'aide d'une seringue S.G.E. de $5 \mu \mathrm{l}$. La colonne spiralée en verre pyrex à $4 \mathrm{~m}$ de longueur, $2 \mathrm{~mm}$ de diamètre interne, et contient comme phase SE-30 à 5 p. 100 .

Support : Supelcoport 100-120 mesh.

La température de l'injecteur et du détecteur est de $250^{\circ} \mathrm{C}$. 


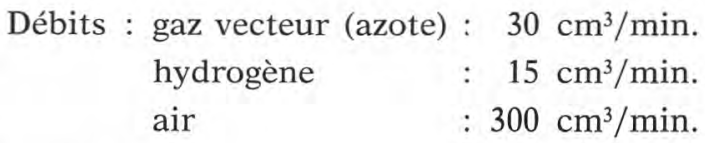

2o PréPARATION DES ÉCHANTILlONS : SILYLATION DES SUCRES

En nous appuyant sur la méthode de Zurcher et al. [22] nous avons mis au point le protocole opératoire suivant :

- prélever $5 \mu \mathrm{l}$ du produit à analyser ;

- évaporer à sec à $80^{\circ} \mathrm{C}$;

- ajouter $50 \mu \mathrm{l}$ de pyridine et $100 \mu \mathrm{l}$ de bis-(triméthylsilyl)-trifluorocétamide (B.S.T.F.A.) à 1 p. 100 de triméthylchlorosilane (T.M.C.S.) ;

- porter à l'étuve $4 \mathrm{~h}$ à $80^{\circ} \mathrm{C}$.

L'échantillon peut se conserver au maximum 4 semaines à $4^{\circ} \mathrm{C}$.

3o AnAlyse :

- température du four : programmation de $2^{\circ} \mathrm{C} / \mathrm{min}$ de $140^{\circ} \mathrm{C}$ à $240^{\circ} \mathrm{C}$;

- injection de 1 à 2 ul de l'échantillon ;

- la durée de la chromatographie est de $85 \mathrm{~min}$.

\section{RESULTATS ET DISCUSSION}

\section{Résultats de la chromatographie en phase gazeuse (CPG)}

La CPG des sucres est une technique couramment admise et comme le montre la figure 1, elle permet un dosage précis de tous les sucres et même de leurs isomères, puisque les formes $\alpha, \beta$ et $\gamma$ sont bien identifiées. De plus, de par sa grande sensibilité et sa sélectivité, la CPG révèle la présence ou non d'autres constituants tels que certains oligosaccharides qui peuvent apparaître dans les hydrolysats. Par contre sa complexité en son temps d'analyse de l'ordre de $6 \mathrm{~h}$ font qu'elle est difficilement applicable dans le cadre d'un contrôle industriel rapide.

\section{Utilisation de la chromatographie liquide à haute performance (CLHP)}

$1^{\circ}$ CHOIX DES CONDITIONS OPÉRATOIRES

La séparation du glucose et du galactose est difficile par CLHP. Ces deux monosaccharides possèdent en effet des structures spatiales très proches l'une de l'autre. Une séparation partielle est obtenue avec un mélange acétonitrile-eau $(87,5-12,5)$ et un débit fixé à $2 \mathrm{ml} / \mathrm{min}$. 


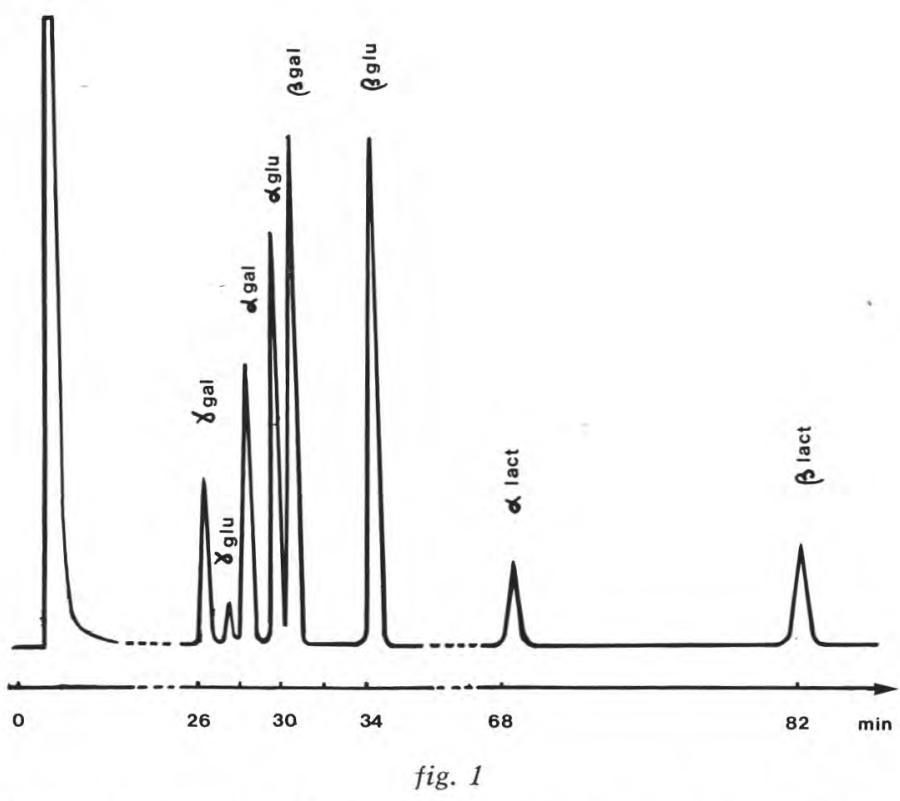

Chromatogramme (CPG) d'un mélange glucose, galactose, lactose. Conditions d'analyse : colonne SE-30 à $5 \mathrm{p} .100$; température du four : programmation de température de $2^{\circ} \mathrm{C} / \mathrm{min}$ de $140^{\circ} \mathrm{C}$ à $240^{\circ} \mathrm{C}$.

De plus, l'analyse du mélange (glucose, galactose, lactose) effectuée dans ces conditions conduit à une élution du lactose qui est très retardée et très lente. Ce phénomène a pour conséquence l'apparition sur le chromatogramme (fig. 2) d'un " pic représentatif » qui, en s'étirant, exclut toute interprétation quantitative précise.

Le faible pouvoir éluant du solvant vis-à-vis du lactose s'explique bien par la faible proportion du constituant le plus polaire c'est-àdire l'eau, dans le mélange acétonitrile-eau (87,5-12,5).

En fait, la séparation du glucose et du galactose n'apparaît pas indispensable et même peut être inutile.

Nous avons donc choisi des modalités opératoires conduisant à la superposition rigoureuse du glucose-galactose et à une durée courte pour l'élution du lactose : colonne chromatographique $(25 \times 4,6 \mathrm{~mm})$ de $5 \mu$; mélange éluant acétonitrile-eau (73-27) ; débit de l'éluant $2,5 \mathrm{ml} / \mathrm{min}$.

Dans ces conditions, l'analyse d'un hydrolysat de lactose pur ou d'un hydrolysat de lactose de lactosérum déprotéiné est possible 


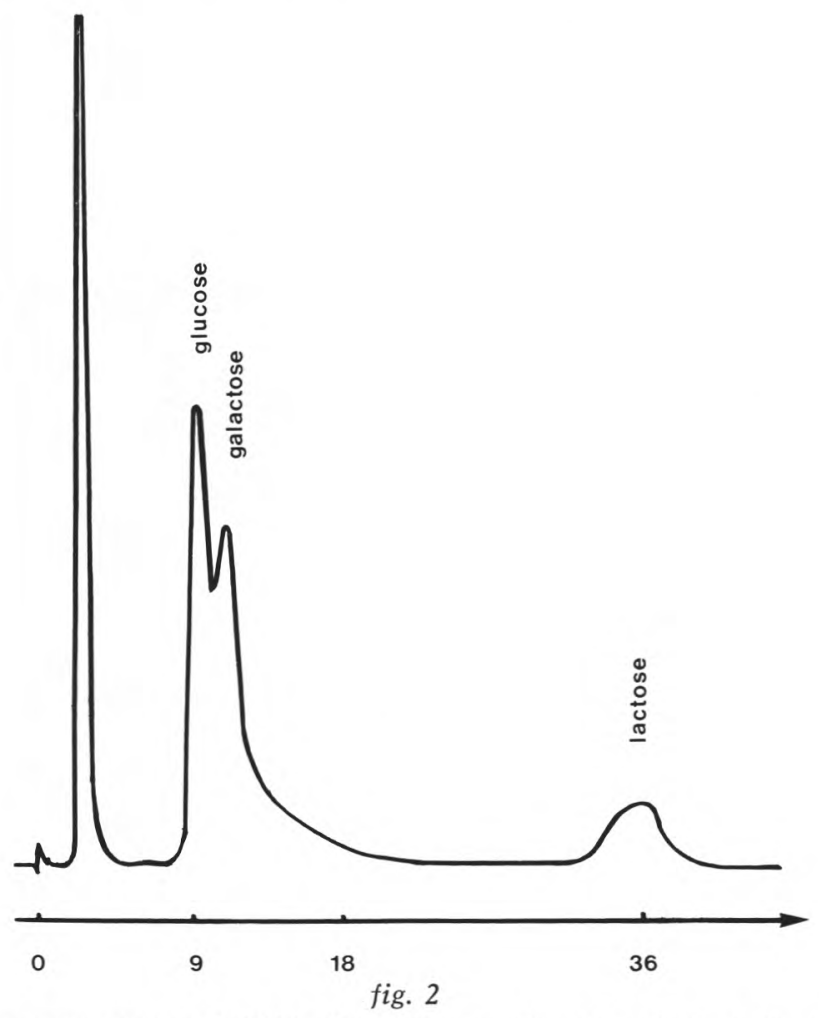

Chromatogramme (CLHP) d'un mélange glucose, galactose, lactose. Colonne : $5 \mu$ Lichrosorb $\mathrm{NH}_{2}$; mélange éluant $\mathrm{CH}_{3} \mathrm{CN} / \mathrm{H}_{2} \mathrm{O}$ $(87,5-12,5 \mathrm{~V} / \mathrm{V})$; débit : $2 \mathrm{ml} / \mathrm{min}$.

en 10 min puisque le temps de préparation se limite à l'addition de l'étalon interne avec, si nécessaire, dilution de l'échantillon.

Toutefois, la comparaison des chromatogrammes obtenus par CLHP (fig. 3) et par CPG (fig. 1) met en évidence la limite de la méthode sur le plan qualitatif : le dosage des isomères est exclu en CLHP.

\section{AsPECT QUANTITATIF}

Le procédé est intéressant sur le plan quantitatif, puisque le nombre réduit de molécules à analyser : trois, dont deux se superposent chromatographiquement, le galactose et le glucose, rend l'utilisation d'un étalon interne possible. Et, comme l'a démontré Black [18], la quantification en CLHP est meilleure avec un étalon interne de réfé- 


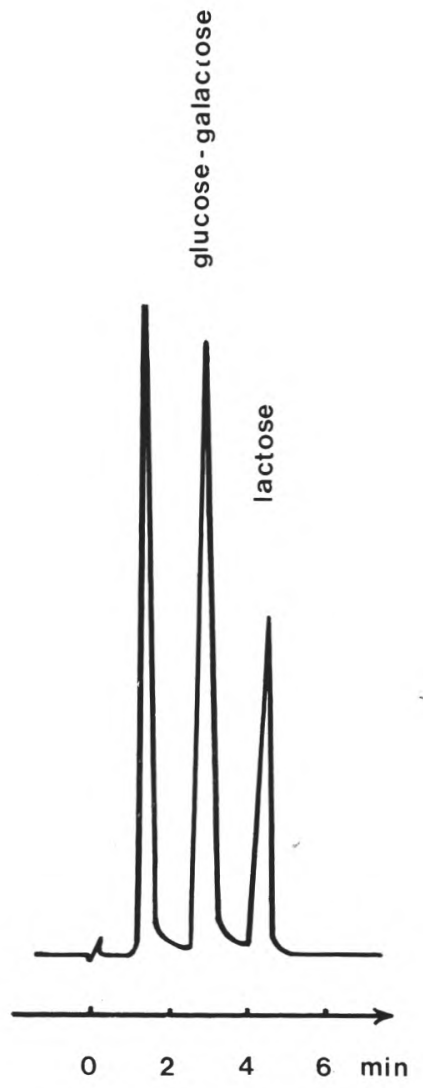

fig. 3

Chromatogramme (CLHP) d'un mélange glucose, galactose, lactose.

Colonne : $5 \mu$ Lichrosorb $\mathrm{NH}_{2}$; mélange éluant $\mathrm{CH}_{3} \mathrm{CN} / \mathrm{H}_{2} \mathrm{O}(73-27 \mathrm{~V} / \mathrm{V})$; débit : $2,5 \mathrm{ml} / \mathrm{min}$.

rence que par simples injections répétitives. A cet effet, nous avons donc choisi le raffinose, un triholoside qui est élué après le lactose (fig. 4).

Le dosage quantitatif s'effectue par l'intégration des surfaces des pics chromatographiques et à l'aide des courbes étalons. L'utilisation du raffinose comme étalon interne permet de tracer des courbes du type $R_{s}=f(c)$ a avec :

$$
\begin{aligned}
\mathrm{R}_{\mathrm{s}} & =\frac{\text { Surface du sucre }}{\text { Surface de l'étalon interne }} \\
\text { et } \mathrm{C} & =\text { concentration en } \mathrm{g} / \mathrm{l} \text { du sucre. }
\end{aligned}
$$

Ces courbes montrent que la réponse du détecteur reste linéaire pour les échelles de concentrations choisies, qui varient de 0 à 5 p. 100 


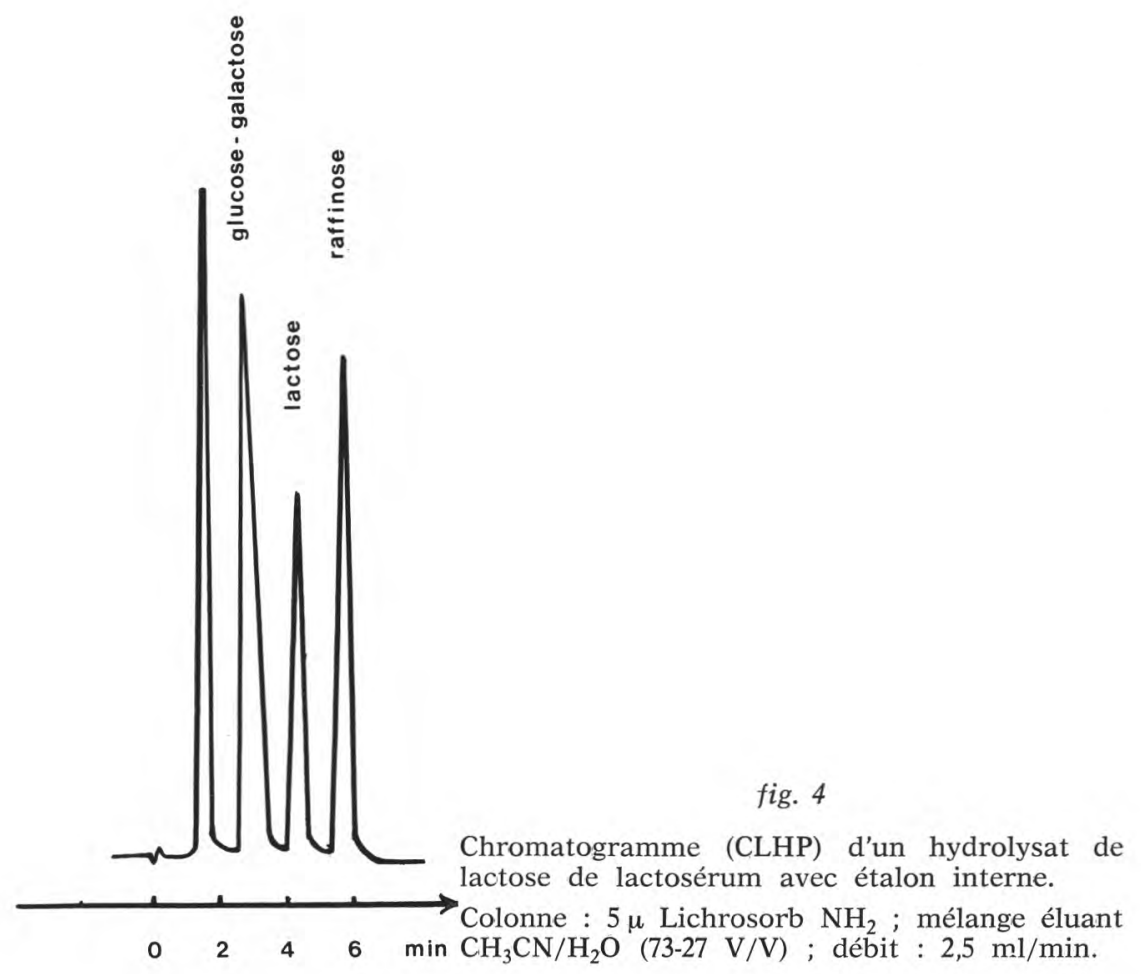

(poids/volume) pour le mélange glucose + galactose et de 0 à 2,5 p. 100 pour le lactose (fig. 5 et 6 ).

De plus, comme on le remarque sur la figure 7, la courbe d'étalonnage du glucose est identique à celle du galactose. De ce fait, même si le mélange de ces deux sucres dans l'hydrolysat analysé n'est pas rigoureusement équimolaire, ce qui est fréquemment observé, le dosage quantitatif du mélange demeure parfaitement valable.

Les résultats quantitatifs sont résumés dans le tableau 1. Ils montrent que la précision est satisfaisante puisque si l'on compare les deux méthodes d'analyse (CLHP et CPG), on observe un écart moyen du taux d'hydrolyse voisin de 2 p. 100.

La répétabilité est également satisfaisante, comme l'indiquent les écarts types, calculés sur 10 essais pour chaque échantillon.

De plus, le tableau 1 met en évidence que la méthode peut s'appliquer aisément aux hydrolysats de lactosérum non déprotéiné. Il suffit alors d'opérer une extraction protéique en traitant l'échantillon par 


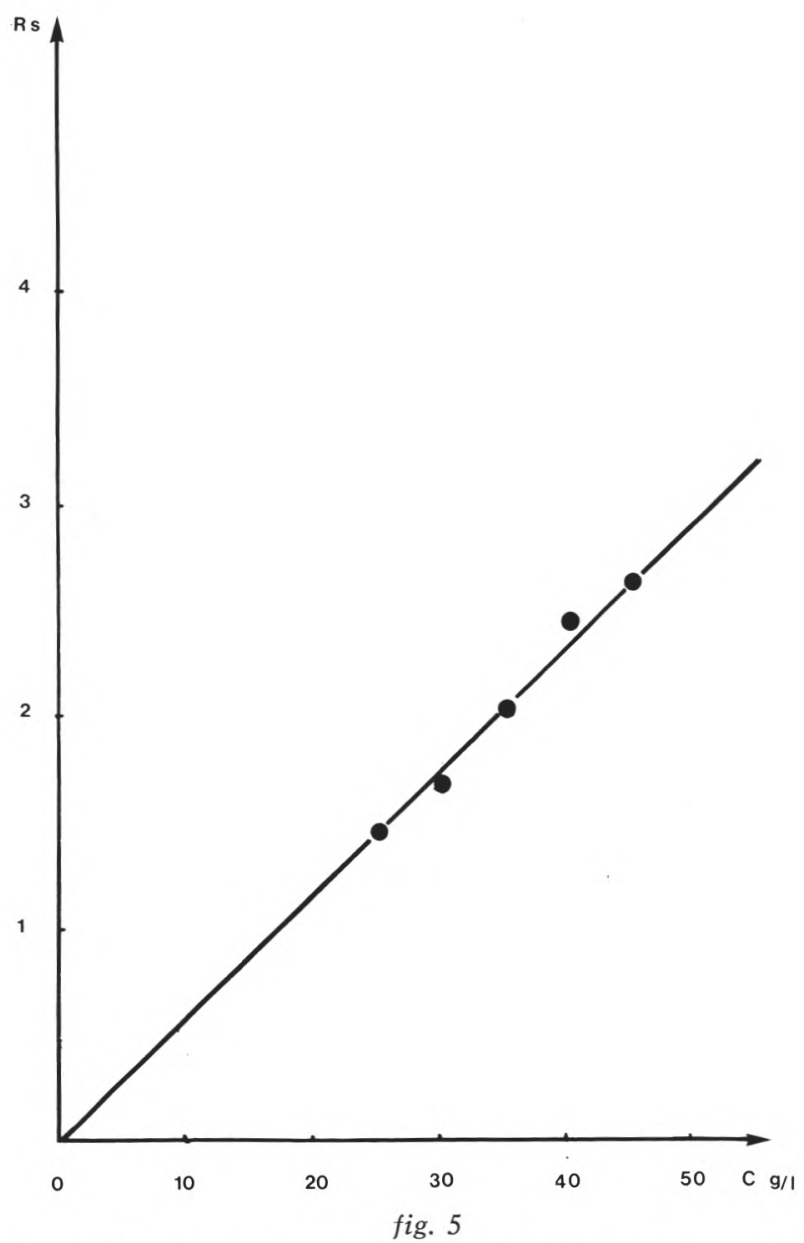

Courbe d'étalonnage du mélange équimolaire glucose-galactose par CLHP.

Colonne : $5 \mu$ Lichrosorb $\mathrm{NH}_{2}$; mélange éluant $\mathrm{CH}_{3} \mathrm{CN} / \mathrm{H}_{2} \mathrm{O}$ $(73-27 \mathrm{~V} / \mathrm{V})$; débit : $2,5 \mathrm{ml} / \mathrm{min}$.

l'acide trichloracétique. Cependant comme le précise Euber et Brunner [6], il est nécessaire dans ce cas d'établir les courbes d'étalonnage des sucres en milieu trichloracétique à 6 p. 100, du fait de l'interférence de ce dernier sur la hauteur des pics représentatifs des glucides. Le temps total pour ce type d'analyse est de l'ordre de $20 \mathrm{~min}$.

Signalons enfin, que l'efficacité de la colonne chromatographique n'est pas affectée avant 2 à 3 mois d'utilisation en routine. 
TABLEAU 1

Détermination du taux d'hydrolyse du lactose du lactosérum déprotéiné ou non par CLHP et par CPG

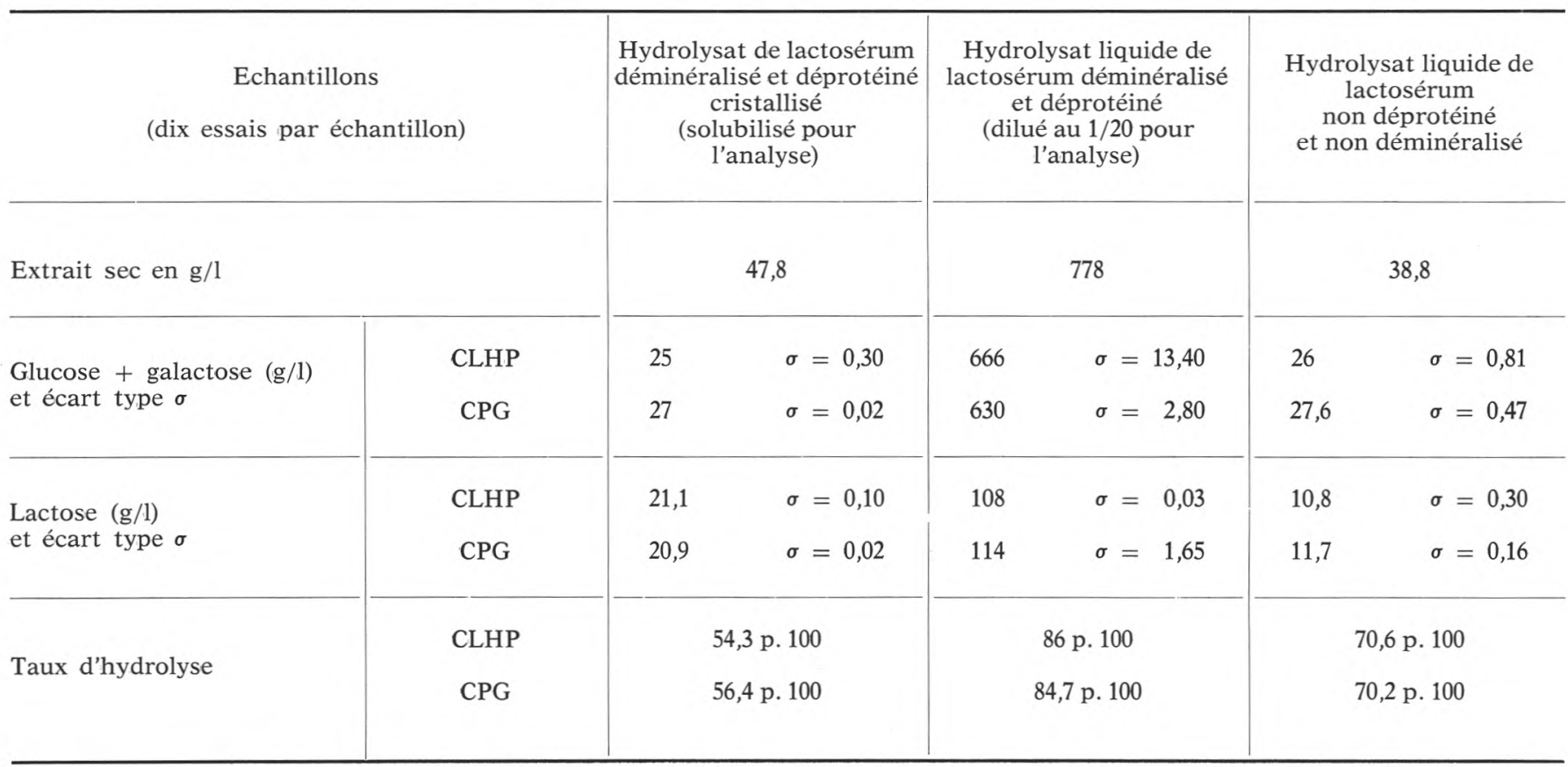




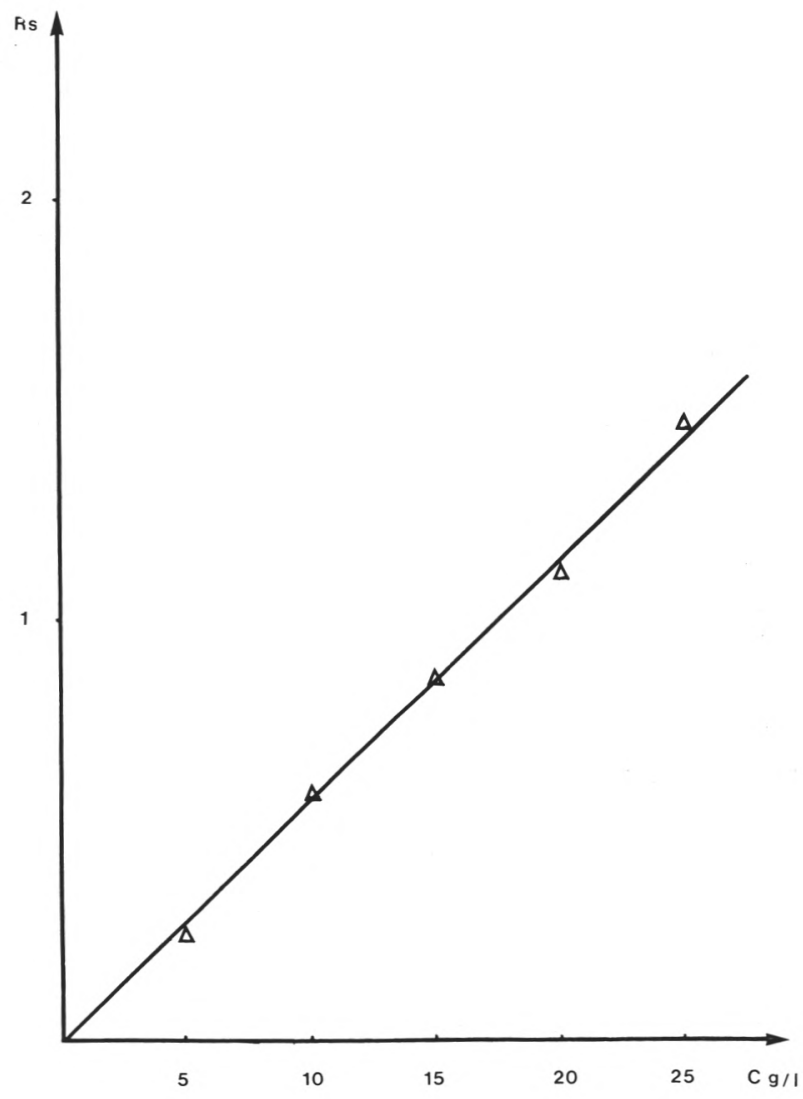

fig. 6

Courbe d'étalonnage du lactose par CLHP.

Colonne : $5 \mu$ Lichrosorb $\mathrm{NH}_{2}$; mélange éluant $\mathrm{CH}_{3} \mathrm{CN} / \mathrm{H}_{2} \mathrm{O}$ $(73-27 \mathrm{~V} / \mathrm{V})$; débit : $2,5 \mathrm{ml} / \mathrm{min}$.

\section{CONCLUSION}

La chromatographie liquide à haute pression permet la détermination rapide du taux d'hydrolyse du lactose.

Le temps d'analyse d'un hydrolysat de lactose pur ou d'un hydrolisat de lactose de lactosérum déprotéiné est de $10 \mathrm{~min}$ et celui d'un hydrolysat de lactose de lactosérum non déprotéiné de $20 \mathrm{~min}$. 


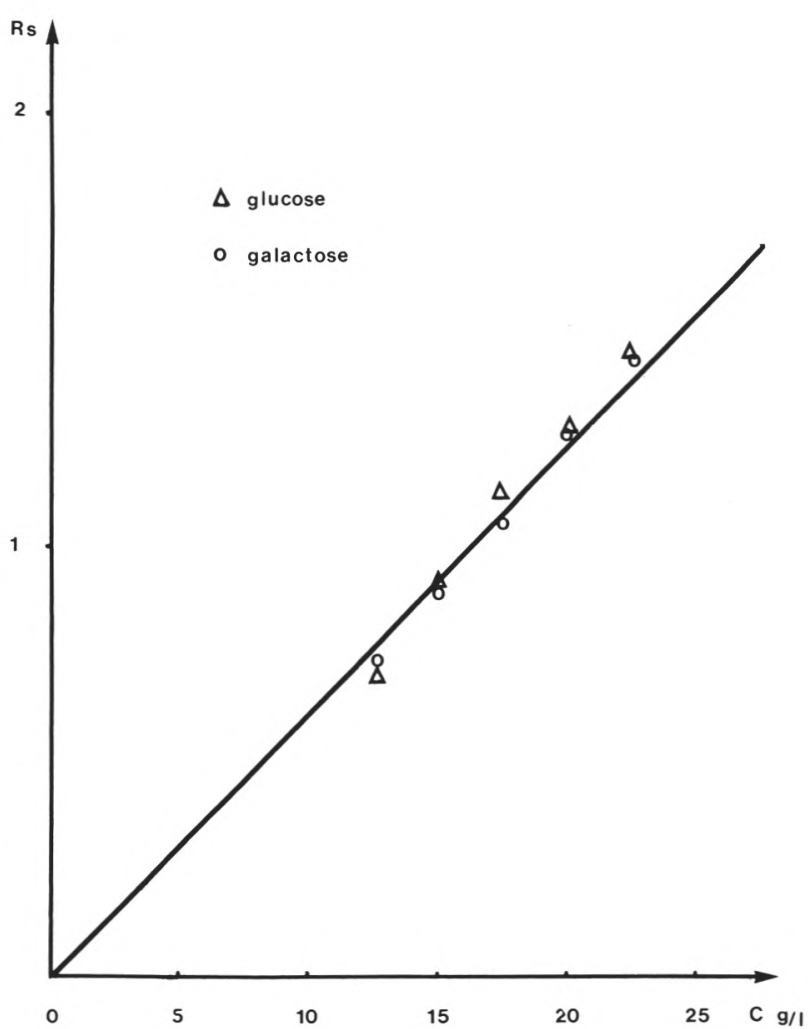

fig. 7

Courbes d'étalonnages du glucose et du galactose par CLHP. Colonne : $5 \mu$ Lichrosorb $\mathrm{NH}_{2}$; mélange éluant $\mathrm{CH}_{3} \mathrm{CN} / \mathrm{H}_{2} \mathrm{O}$ $(73-27 \mathrm{~V} / \mathrm{V})$; débit : $2,5 \mathrm{ml} / \mathrm{min}$.

Le glucose et le galactose ne sont pas séparés et sont dosés sous la forme d'un seul pic chromatographique.

Le raffinose est utilisé comme étalon interne, ce qui conduit à une précision du même ordre que celle obtenue par chromatographie en phase gazeuse.

Cette méthode sélective et rapide est donc facilement utilisable. Elle est en outre, spécialement adaptée aux contrôles industriels. 


\section{Remerciements}

Les auteurs remercient Mlle Lebouteiller et Mme Anne Navaro de leurs collaborations techniques.

\section{S um m a r y}

A QUICK AND SPECIFIC DETERMINATION OF THE RATE OF LACTOSE HYDROLYSIS BY HIGH PERFORMANCE LIQUID CHROMATOGRAPHY

USING AN INTERNAL STANDARD

High performance liquid chromatography enables a quick determination of the rate of lactose hydrolysis.

Only ten (10) minutes are necessary to analyse a pure lactose hydrolysate or a hydrolysate of lactose from deproteinated whey and twenty (20) minutes for a hydrolysate of lactose from non deproteinated whey.

Glucose and galactose are not separated and are determined together.

The use of raffinose as internal standard results in an accuracy similar to the one obtained through gas liquid chromatography.

This selective and quick method can be used by any analysis laboratory and is especially suited to industrial controls.

Reçu pour publication en novembre 1980.

\section{Bibliographie}

[1] Black (L. T.) and Bagley (E. B.) (1978). - Determination of oligo-saccharides in soybeans by high pressure liquid chromatography using an internal standard. J. Amer. Oil. Chem. Soc., 55, 228-232.

[2] Boemringer-Mannheim (1977). - Méthodes d'analyses enzymatiques en chimie alimentaire.

[3] Conrad (E. C.) and Palmer (J. K.) (1976). - Rapid analysis of carbohydrates by high pressure liquid Chromatography. Food Technology, october, 84-92.

[4] Demaimay (M.) (1977). - Etude comparative des produits d'hydrolyse enzymatique et catalytique du lactosérum déprotéiné sur résines échangeuses d'ions. Le Lait, 57, 351-361.

[5] de Vries (J. W.), Heroff (J. C.) and Egberg (D. C.) (1979). - High pressure liquid chromatographic determination of carbohydrates in food products: Evaluation of method. J. Assoc. Off. Anal. Chem., 62, 1292-1296.

[6] Euber (J. R.) and BRunNer (J. R.) (1979). - Determination of lactose in milk products by high performance liquid chromatography. J. Dairy Sci., 62, 685-690.

[7] HagGET (T. O. R.) (1976). - The acid catalysed hydrolysis of lactose using a cation exchange resin. N.Z.I. Dairy Sci. Technol., 11, 176-179. 
[8] Harju (M.), Heikonen (M.), Kreula (M.), Viskari (R.) and Linko (M.) (1978). - Hydrolysis of whey with immobilized lactase. First European Congress in Biotechnology. Interlaken Pub. 2, 131-132.

[9] Hurst (W. J.), Martin (R. A.) and Zoumas (B. L.). (1979), - Application of HPLC to characterization of individual carbohydrates in foods. J. Food Science, 44, 892-895.

[10] Merck-Darmstadt. - Détermination photométrique de la concentration en glucose. Documentation technique.

[11] Moll (M.), Bazard (D.) et Flayeux (R.) (1978) . - Dosage des sucres fermentescibles dans le moût par chromatographie liquide à haute performance (CLHP). Bios., 9, 23-26.

[12] Mouillet (L.), Luquet (E. M.) et Boudier (J. F.) (1977). - Détermination des sucres par chromatographie en phase gazeuse. Le Lait, 57, 561-562, 37-54.

[13] Nujpels (H. H.) (1977). - Maxilact lactase in dairy industry. North European Dairy J., 10, 358-360 ; 11-12, 382-385.

[14] Olson (N.F.) and Richardson (T.) (1974). - Immobilized enzymes in food processing and analysis. J. Food Science, 39, 653-659.

[15] Pechanek (U.), Blaicher (G.), Pfannhauser (W.) and Woidich (H.) (1980). Application of column liquid chromatography (HPLC) to special problems in food chemistry. A laboratory note. Chromatographia, 13, 421-427.

[16] Ramet (J. P.), NovaK (G.), Evers (P. A.) et NiJpels (H. H.) (1979). - Application de la cryométrie à la mesure de l'hydrolyse enzymatique du lactose. Le Lait, 59, 581-582, 46-53.

[17] Roger (L.), Thapon (J. L.), Maubois (J. L.) et Brulé (G.) (1976). - Hydrolyse du lactose contenu dans l'ultrafiltrat de lait ou de lactosérum en réacteur enzymatique à membrane. Le Lait, 56, 551-552, 56-75.

[18] Tauber (H.) and Kleiner (I.) (1932). - A method for the determination of monosaccharides in the presence of oligosaccharides and its application to blood analysis. J. Biol. Chem., 99, 249-255.

[19] Thompson (M. P.) and Brower (D. P.) (1974). - Manufacture of Cheddar cheese from hydrolyzed lactose milk. J. Dairy Sci., 57, 598.

[20] Ugrinovits (M.) (1980). - Zuckeranalyse mit GC, HPLC, DC und enzymatisch - Ein Vergleich der Methoden. Chromatographia, 13, 386-394.

[21] Warthesen (I. I.) and Kramer (P. L.) (1979). - Analysis of sugars in milk and ice cream by high pressure liquid chromatography. J. Food Science, $44,626-627$.

[22] Zurcher (K.), Hadorn (H.) und Strack (Ch.) (1975). - Gaschromatographische Zurkerbestimmung. Mitt. Gebiete Lebensm. Hyg., 66, 92-116. 